\title{
Structural System Identification with Extended Kalman Filter and Orthogonal Decomposition of Excitation
}

\author{
Y. Ding, ${ }^{1,2}$ B. Y. Zhao, ${ }^{1,2}$ and B. Wu ${ }^{1,2}$ \\ ${ }^{1}$ Key Lab of Structures Dynamic Behavior and Control (Harbin Institute of Technology), Ministry of Education, \\ Heilongjiang, Harbin 150090, China \\ ${ }^{2}$ School of Civil Engineering, Harbin Institute of Technology, Harbin 150090, China \\ Correspondence should be addressed to Y. Ding; dingyong@hit.edu.cn
}

Received 10 November 2013; Accepted 25 December 2013; Published 17 February 2014

Academic Editor: Xiao-Wei Ye

Copyright ( $\odot 2014$ Y. Ding et al. This is an open access article distributed under the Creative Commons Attribution License, which permits unrestricted use, distribution, and reproduction in any medium, provided the original work is properly cited.

\begin{abstract}
Both the structural parameter and external excitation have coupling influence on structural response. A new system identification method in time domain is proposed to simultaneously evaluate structural parameter and external excitation. The method can be used for linear and hysteresis nonlinear structural condition assessment based on incomplete structural responses. In this method, the structural excitation is decomposed by orthogonal approximation. With this approximation, the strongly time-variant excitation identification is transformed to gentle time-variant, even constant parameters identification. Then the extended Kalman filter is applied to simultaneously identify state vector including the structural parameters and excitation orthogonal parameters in state space based on incomplete measurements. The proposed method is validated numerically with the simulation of threestory linear and nonlinear structures subject to external force. The external force on the top floor and the structural parameters are simultaneously identified with the proposed system identification method. Results from both simulations indicate that the proposed method is capable of identifing the dynamic load and structural parameters fairly accurately with contaminated incomplete measurement for both of the linear and nonlinear structural systems.
\end{abstract}

\section{Introduction}

The evaluation of the structural parameter and external excitation are two main parts of structural health monitoring, which contribute efficiently to structural maintenance and management. After the severe external excitation, infrastructures always suffer from structural damages and some components may perform nonlinearly. The nonlinearities in structural system may have adversely influence on structural health monitoring (SHM) and structural control. Both the structural parameter and external excitation have coupling influence on structural response. Therefore, the evaluation of one aspect without considering another aspect may cause error in the identification result. Exact knowledge of structural parameter and the excitation time history are essential to the rapid postevent structural condition assessment and the prediction of load-bearing capacity.

Vibration-based SHM methods are investigated actively for decades [1-3]. An extensive summary of health monitoring and vibration-based structural condition assessment of civil engineering structures are provided $[1,4]$. Zou et al. [5] summarized the methods on vibration-based damage detection and health monitoring for composite structures, especially in delamination modelling techniques and delamination detection. More recently, Chen and $\mathrm{Li}$ [6] presented methods to identify structural parameters and input time history from output-only measurements iteratively. Lu and Law [7] identify the physical parameters and the input excitation force of linear structures based on the sensitivities of dynamic response. Law and Yong [8] proposed substructural condition assessment method for structural damage detection and external force identification of linear structural system. Online identification methods for linear substructures have been developed by Ding [9] and the local damage can be accurately identified. Sun and Betti [10] proposed a simultaneous identification method for linear structure with artificial bee colony algorithm. Lei et al. [11] successfully identified the shear building based on partial output measurements with EKF and least-squares estimator. 
However, the target structures investigated above are almost linear and time-invariant.

In the past few decades, numerous methods have been developed for structural model updating and external excitation of nonlinear structures. The Kalman filter is an effective means of parameter identification and input estimation for a linear or nonlinear structure. Two forms of the extended recursive least-squares algorithm were considered for the identification of system parameter and the tracking of a chirped sinusoid with additive noise [12]. Other timevariant parameter identification methods are the online identification of nonlinear hysteretic structure with an adaptive tracking techniques based on least-squares estimation [13], nonlinear normal mode analysis which considered the nonlinearity of structural system [14-16], an online sequential weighted least-squares support vector machine technique to quantify the structural parameter changes when the measurement involves damage events [17], and an adaptive tracking technique based on extended Kalman filter for identifying the structural parameters and their increments [18]. These methods do not require a priori knowledge of the time of occurrence of the anomalies. Hence, these methods could be applied to conduct the structural condition assessment online. However, most existing methods for timevariant parameter identification may require the complete measurement of structural response.

In this paper, a simultaneous identification method for structural parameter and external excitation is proposed for both linear and nonlinear structures based on the extended Kalman filter. The structural parameters include stiffness, damping, and the parameters of nonlinear model. In this study, the excitation time history is decomposed by orthogonal polynomial. The coefficients of orthogonal polynomial and structural parameter are taken as state variables. With extended Kalman filter the state vector is identified recursively. The proposed method was validated numerically with the simulation of a linear shear frame and a hysteretic nonlinear shear frame. Results from the numerical simulations indicate that the proposed method can be used to identify structural parameter and external excitations effectively based on incomplete contaminated structural responses measurements.

\section{Equation of Motion in State Space}

The equation of motion of an $N$ dofs linear structural system subject to external excitation is

$$
\mathbf{M} \ddot{\mathbf{x}}(t)+\mathbf{C} \dot{\mathbf{x}}(t)+\mathbf{K x}(t)=\mathbf{L F},
$$

where $\mathbf{M}, \mathbf{C}$, and $\mathbf{K}$ are the mass, damping, and stiffness matrices of the structural system, respectively. $\mathbf{F}$ is the external force acting on the structure and $\mathbf{L}$ is the location matrix of external force. $\ddot{\mathbf{x}}, \dot{\mathbf{x}}$, and $\mathbf{x}$ are, respectively, the vectors of acceleration, velocity, and displacement responses of the structural system. The equation of motion of the linear structural system shown in (1) can also be expressed in the state space generally as the following equation:

$$
\dot{\mathbf{Z}}(t)=\mathbf{A Z}(t)+\mathbf{B L} \times \mathbf{F}(t),
$$

where

$$
\mathbf{Z}=\left[\begin{array}{c}
\mathbf{x} \\
\dot{\mathbf{x}}
\end{array}\right], \quad \mathbf{A}=\left[\begin{array}{cc}
0 & \mathbf{I} \\
-\mathbf{M}^{-1} \mathbf{K} & -\mathbf{M}^{-1} \mathbf{C}
\end{array}\right], \quad \mathbf{B}=\left[\begin{array}{c}
0 \\
\mathbf{M}^{-1}
\end{array}\right] .
$$

The equation of motion of a hysteretic nonlinear structural system subject to excitation can be written as

$$
\mathbf{M} \ddot{\mathbf{x}}(t)+\mathbf{C} \dot{\mathbf{x}}(t)+\mathbf{K z}(t)=\mathbf{L F}(t),
$$

where $\mathbf{z}(t)=\left[\begin{array}{llll}\mathbf{z}_{\mathbf{1}}(t) & \mathbf{z}_{\mathbf{2}}(t) & \cdots & \mathbf{z}_{\mathbf{i}}(t)\end{array}\right]^{T}$ is the hysteretic component vector, and the hysteretic component can be represented as

$$
\dot{z}_{i}=\dot{x}_{i}-\beta_{i}\left|\dot{x}_{i}\right|\left|z_{i}\right|^{\alpha_{i}-1} z_{i}-\gamma_{i} \dot{x}_{i}\left|z_{i}\right|^{\alpha_{i}}
$$

where subscript $i$ represent the $i$ th story. $\dot{x}_{i}$ and $z_{i}$ are, respectively, the $i$ th story velocity and hysteretic component. $\beta_{i}$ and $\gamma_{i}$ are nondimensional parameters which decide the shape of the hysteresis loop of the $i$ th story. $\alpha_{i}$ is a nondimensional parameter that controls the smoothness of the hysteresis loop of the $i$ th story. The equation of motion of the nonlinear structural system shown in (5) can also be expressed in the state space generally as the following equation:

$$
\begin{aligned}
\dot{\mathbf{Z}}(t) & =f(\mathbf{Z}, \mathbf{F}, t) \\
& =\left[\begin{array}{c}
\dot{\mathbf{x}}(t) \\
\mathbf{M}^{-1}[\mathbf{L F}(t)-(\mathbf{C X}(t)+\mathbf{K Z}(t))] \\
\dot{\mathbf{x}}(t)-\boldsymbol{\beta}|\dot{\mathbf{x}}(t)||\mathbf{z}(t)|^{\boldsymbol{\alpha}-\mathbf{1}} \mathbf{z}-\boldsymbol{\gamma} \dot{\mathbf{x}}(t)|\mathbf{z}(t)|^{\boldsymbol{\alpha}}
\end{array}\right],
\end{aligned}
$$

where $f(\mathbf{Z}, \mathbf{F}, t)$ is a nonlinear function of $\mathbf{Z}$ in state space. For the linear and nonlinear structures, responses can be recursively calculated by (4) or (6), respectively. The identification method proposed in this paper is based on these two types of state space equations.

\section{Extended Kalman Filter for Structural System Identification}

The equation of motion of an $N$ dofs structure under load excitation can be written as

$$
\mathbf{M} \ddot{\mathbf{x}}(t)+\mathbf{F}_{c}[\dot{\mathbf{x}}(t)]+\mathbf{F}_{s}[\mathbf{x}(t)]=\mathbf{L F}(t),
$$

where $\mathbf{F}_{c}[\dot{\mathbf{x}}(t)]$ denotes damping force vector and $\mathbf{F}_{s}[\mathbf{x}(t)]$ denotes stiffness force vector. Introducing an extended state vector with a dimension of $2 n+m$,

$$
\mathbf{Z}(t)=[\mathbf{x}(t), \dot{\mathbf{x}}(t), \boldsymbol{\theta}]^{\mathbf{T}},
$$

where $n$ denote the dimension of original state vector and $\boldsymbol{\theta}=\left[\theta_{1}, \theta_{2}, \ldots, \theta_{m}\right]^{T}$ denote $m$-unknown parameter of the structure, including damping, stiffness, or nonlinear parameters. Equation (8) can be written in continuous state space as

$$
\dot{\mathbf{Z}}(t)=f(\mathbf{Z}, \mathbf{F}, t)+\mathbf{w}(t)
$$


where $\mathbf{Z}, \mathbf{F}$, and $\mathbf{w}$ are the state, the external force, and the process noise vectors which are assumed to be zero-mean Gaussian noise. The observation equation at discrete time steps $t_{k}=k \Delta t$ can be written as

$$
\mathbf{y}\left(t_{k}\right)=h\left(\mathbf{Z}\left(t_{k}\right)\right)+\mathbf{v}\left(t_{k}\right)
$$

where $\mathbf{y}\left(t_{k}\right)$ and $\mathbf{v}\left(t_{k}\right)$ are the measurement response and measurement noise vectors. Both process and measurement noises are assumed to be uncorrelated zero-mean Gaussian random processes. With the EKF, (9) and (10) can be linearized as

$$
\begin{gathered}
f(\mathbf{Z}, \mathbf{F}, t) \approx f\left(\widehat{\mathbf{Z}}_{k \mid k}, \mathbf{F}, t\right)+\mathbf{A}_{k \mid k}\left(\mathbf{Z}-\widehat{\mathbf{Z}}_{k \mid k}\right), \\
h\left(\mathbf{Z}_{k+1}\right) \approx h\left(\widehat{\mathbf{Z}}_{k+1 \mid k}\right)+\mathbf{H}_{k+1 \mid k}\left(\mathbf{Z}_{k+1}-\widehat{\mathbf{Z}}_{k+1 \mid k}\right),
\end{gathered}
$$

in which $\mathbf{A}_{k \mid k}$ is the linearized matrix of $f\left(\widehat{\mathbf{Z}}_{k \mid k}, \mathbf{F}, t\right)$ and $\mathbf{H}_{k+1 \mid k}$ is the linearized matrix of $h\left(\widehat{\mathbf{Z}}_{k+1 \mid k}\right)$ as follows:

$$
\begin{aligned}
\mathbf{A}_{k \mid k} & =\left[\frac{\partial f}{\partial \mathbf{Z}}\right]_{\widehat{Z}_{k \mid k}}, \\
\mathbf{H}_{k+1 \mid k} & =\left[\frac{\partial h}{\partial \mathbf{Z}}\right]_{\widehat{Z}_{k+1 \mid k}} .
\end{aligned}
$$

Equation (11) constitutes a classical system with continuous time evolution of states and discrete measurements. The hybrid EKF can estimate the states of $\mathbf{Z}((k+1) \Delta t)$ at $t=k \Delta t$ from the contaminated measurements through the following set of equations:

$$
\widehat{\mathbf{Z}}_{k+1 \mid k}=\widehat{\mathbf{Z}}_{k \mid k}+\int_{k \Delta t}^{(k+1) \Delta t} f\left(\widehat{\mathbf{Z}}_{t \mid k}\right) d t .
$$

And the recursive optimal solution for $\mathbf{Z}((k+1) \Delta t)$ at $t=$ $(k+1) \Delta t$ is shown as follows:

$$
\widehat{\mathbf{Z}}_{k+1 \mid k+1}=\widehat{\mathbf{Z}}_{k+1 \mid k}+\mathbf{K}_{k+1}\left[\mathbf{Y}_{k+1}-h\left(\widehat{\mathbf{Z}}_{k+1 \mid k}\right)\right],
$$

where $\mathbf{K}_{k+1}$ is the Kalman gain matrix:

$$
\mathbf{K}_{k+1}=\mathbf{P}_{k+1 \mid k} \mathbf{H}_{k+1 \mid k}^{T}\left[\mathbf{H}_{k+1 \mid k} \mathbf{P}_{k+1 \mid k} \mathbf{H}_{k+1 \mid k}^{T}+\mathbf{R}_{k+1}\right]^{-1},
$$

where $\mathbf{P}_{k+1 \mid k}$ is the error covariance matrix of a posteriori state vector $\widehat{\mathbf{Z}}_{k+1 \mid k}$ :

$$
\mathbf{P}_{k+1 \mid k}=\boldsymbol{\Phi}_{k+1 \mid k} \mathbf{P}_{k \mid k} \boldsymbol{\Phi}_{k+1 \mid k}^{T}+\mathbf{Q}_{k},
$$

where $\mathbf{Q}_{k}$ is the covariance matrix of process noise at $t=$ $k \Delta t . \Phi_{k+1 \mid k}$ is the state transition matrix of the linearized system given by

$$
\Phi_{k+1 \mid k} \approx \mathbf{I}+\Delta t \times \mathbf{A}_{k \mid k}
$$

in which I is the unit matrix of dimension $2 n+m . \mathbf{P}_{k \mid k}$ in (17) is the error covariance matrix of a priori state vector $\widehat{\mathbf{Z}}_{k+1 \mid k}$ given by $\boldsymbol{\Phi}_{k+1 \mid k} \approx \mathbf{I}+\Delta t \times \mathbf{A}_{k \mid k}$ as follows:

$$
\mathbf{P}_{k \mid k}=\left[\mathbf{I}-\mathbf{K}_{k} \mathbf{H}_{k \mid k-1}\right] \mathbf{P}_{k \mid k-1}\left[\mathbf{I}-\mathbf{K}_{k} \mathbf{H}_{k \mid k-1}\right]^{T}+\mathbf{K}_{k} \mathbf{R}_{k} \mathbf{K}_{k}^{T} .
$$

\section{Orthogonal Decomposition of Excitation}

The external force is always difficult to be directly identified with extended Kalman filter since it is nonstationary and time-variant. However, the input $F$ is a kind of random process which can be decomposed by standard orthogonal polynomial. Since the orthogonal is constant, the input history will be reconstructed as if the orthogonal parameters can be identified. Therefore, the input identification transforms to the polynomial parameters identification based on input orthogonal decomposition method. The input in (7) can be decomposed as follows:

$$
F^{i}(t)=\sum_{i=1}^{N_{f}} \sum_{m=1}^{N_{m}} w_{m}{ }^{i} T_{m}^{i}(t),
$$

where $w_{m}{ }^{i}$ is the polynomial coefficients of the ith input. $T^{i}$ is the $m$ th orthogonal polynomial of the $i$ th input. $N_{m}$ is the order of input decomposition. $N_{f}$ is the number of inputs. The orthogonal polynomial $T_{m}^{i}$ and order of input decomposition $N_{m}$ can affect the accuracy of input approximation. The order of input decomposition $N_{m}$ is closely related to input history length and complexity. The orthogonal polynomial $T_{m}^{i}$ can be determined in different kinds based on different decomposition methods. The Chebyshev decomposition method is one of the most accuracy methods of random input fitting. The Chebyshev decomposition orthogonal polynomial can be written as follows:

$$
\begin{gathered}
T_{1}=\frac{1}{\sqrt{\pi}}, \\
T_{2}=\sqrt{\frac{2}{\pi}}\left(\frac{2 t}{T}-1\right), \\
T_{n+1}(t)=2\left(\frac{2 t}{T}-1\right) T_{n}(t)-T_{n-1}(t) \\
\left(n=2,3, \ldots, N_{m}-1\right),
\end{gathered}
$$

where $T$ is the length of the input history and $N_{m}$ is the order of decomposition. Based on the Chebyshev standard orthogonal polynomial decomposition, (7) can be written as follows:

$$
\mathbf{M} \ddot{\mathbf{x}}(t)+\mathbf{F}_{c}[\dot{\mathbf{x}}(t)]+\mathbf{F}_{s}[\mathbf{x}(t)]=\sum_{i=1}^{N_{f}} \sum_{m=1}^{N_{m}} w_{m}{ }^{i} T_{m}^{i}(t) .
$$

The input can be reconstructed if the polynomial parameters $w_{m}{ }^{i}$ can be identified.

\section{Simultaneous Identification of Structural System}

A new time domain simultaneous identification method is proposed in this section based on orthogonal decomposition of excitation and extended Kalman filter. The input history is firstly decomposed by the Chebyshev standard orthogonal polynomial as shown in (20). The number of decomposition 
order is based on the input length and complexity. Then, the structural parameters and polynomial parameters will be identified by extended Kalman estimator based on structural measurements, as shown from (12) to (19).

5.1. N-Storey Linear Structure. The equation of motion is given by

$$
\begin{gathered}
\mathbf{M}\left\{\begin{array}{c}
\ddot{x}_{1}(t) \\
\ddot{x}_{2}(t) \\
\vdots \\
\ddot{x}_{n}(t)
\end{array}\right\}+\mathbf{C}\left\{\begin{array}{c}
\dot{x}_{1}(t) \\
\dot{x}_{2}(t) \\
\vdots \\
\dot{x}_{n}(t)
\end{array}\right\}+\mathbf{K}\left\{\begin{array}{c}
x_{1}(t) \\
x_{2}(t) \\
\vdots \\
x_{n}(t)
\end{array}\right\} \\
=\mathbf{L}\left(\sum_{i=1}^{N_{f}} \sum_{m=1}^{N_{m}} w_{m}{ }^{i} T_{m}^{i}(t)\right),
\end{gathered}
$$

where

$$
\begin{gathered}
\mathbf{M}=\left[\begin{array}{llll}
m_{1} & & & \\
& m_{2} & & \\
& & \ddots & \\
& & & m_{n}
\end{array}\right], \\
\mathbf{C}=\left[\begin{array}{cccc}
c_{1}+c_{2} & -c_{2} & \\
-c_{2} & c_{2}+c_{3} & \\
& & \ddots & -c_{n} \\
& & -c_{n} & c_{n}
\end{array}\right], \\
\mathbf{K}=\left[\begin{array}{cccc}
k_{1}+k_{2} & -k_{2} & & \\
-k_{2} & k_{2}+k_{3} & \ddots & -k_{n} \\
& & -k_{n} & k_{n}
\end{array}\right] .
\end{gathered}
$$

Beside $\mathbf{w}=\left[w_{1}, w_{2}, \ldots, w_{i}\right]^{T}, i=\left(1,2, \ldots, N_{f}\right)$, is the polynomial coefficients vector. In (23), $x_{i}(t)$ is the absolute structural response. $T_{m}^{i}$ is the orthogonal polynomial which is given by (21). The state vector is defined as

$$
\mathbf{Z}(t)=\left[x_{1 \sim n}, \dot{x}_{1 \sim n}, k_{1 \sim n}, c_{1 \sim n}, w_{1 \sim N_{m}}\right]^{\mathrm{T}} .
$$

Consider that $\dot{k}_{1 \sim n}=\dot{c}_{1 \sim n}=\dot{w}_{1 \sim N_{m}}=0$. Equation (23) can be written in state space as follows:

$$
\left[\begin{array}{c}
\dot{\mathbf{x}} \\
\ddot{\mathbf{x}} \\
\dot{\mathbf{k}} \\
\dot{\mathbf{c}} \\
\dot{\mathbf{w}}
\end{array}\right]=\left[\begin{array}{c}
\dot{\mathbf{x}} \\
\mathbf{M}^{-\mathbf{1}}\left[\mathbf{L} \sum_{i=1}^{N_{f}} \sum_{m=1}^{N_{m}} w_{m}{ }^{i} T^{i}{ }_{m}-\left(\mathbf{F}_{\mathbf{c}}[\mathbf{c}, \dot{\mathbf{x}}]+\mathbf{F}_{\mathbf{s}}[\mathbf{k}, \mathbf{x}]\right)\right] \\
\mathbf{0} \\
\mathbf{0}
\end{array}\right] .
$$

The measurement can be obtained in the discrete time step from displacements or accelerations. The observation

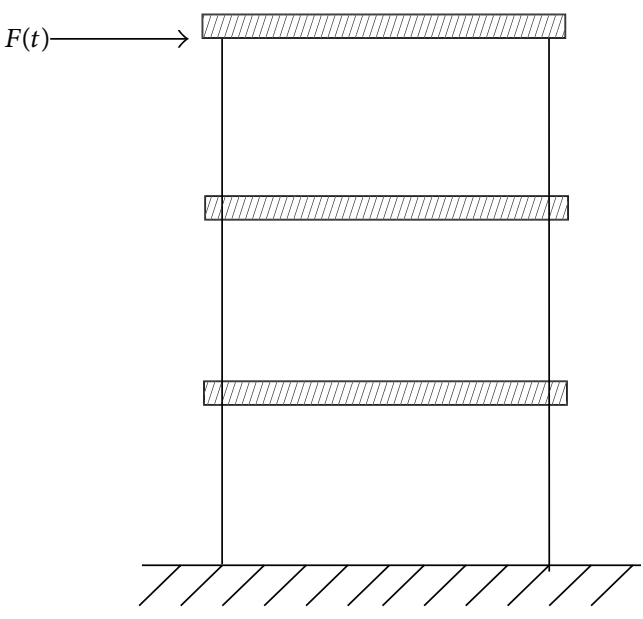

Figure 1: Three-storey frame.

equation can be written with the measurement of the displacement as

$$
\left[\begin{array}{c}
y_{1} \\
y_{2} \\
\vdots \\
y_{n}
\end{array}\right]=\left[\begin{array}{lllll}
\mathbf{I} & \mathbf{0} & \mathbf{0} & \mathbf{0} & \mathbf{0}
\end{array}\right]\left[\begin{array}{c}
\mathbf{x} \\
\dot{\mathbf{x}} \\
\mathbf{k} \\
\mathbf{c} \\
\mathbf{w}
\end{array}\right] .
$$

In which $\mathbf{I}$ is the unit matrix of dimension $n$.

5.2. N-Story Hysteretic Structure. The equation of motion is given by

$$
\begin{gathered}
\mathbf{M}\left\{\begin{array}{c}
\ddot{x}_{1}(t) \\
\ddot{x}_{2}(t) \\
\vdots \\
\ddot{x}_{n}(t)
\end{array}\right\}+\mathbf{C}\left\{\begin{array}{c}
\dot{x}_{1}(t) \\
\dot{x}_{2}(t) \\
\vdots \\
\dot{x}_{n}(t)
\end{array}\right\}+\mathbf{K}\left\{\begin{array}{c}
z_{1}(t) \\
z_{2}(t) \\
\vdots \\
z_{n}(t)
\end{array}\right\} \\
=\mathbf{L}\left(\sum_{i=1}^{N_{f}} \sum_{m=1}^{N_{m}} w_{m}^{i} T_{m}^{i}(t)\right),
\end{gathered}
$$

in which

$$
\mathbf{M}=\left[\begin{array}{cccc}
m_{1} & & & \\
\vdots & m_{2} & & \\
\vdots & \vdots & \ddots & \\
m_{n} & m_{n} & \ldots & m_{n}
\end{array}\right]
$$

$$
\mathbf{C}=\left[\begin{array}{ccccc}
c_{1} & -c_{2} & & & \\
& c_{2} & -c_{3} & & \\
& & \ddots & \ddots & \\
& & & c_{n-1} & -c_{n} \\
& & & & c_{n}
\end{array}\right],
$$

$$
\mathbf{K}=\left[\begin{array}{ccccc}
k_{1} & -k_{2} & & & \\
& k_{2} & -k_{3} & & \\
& & \ddots & \ddots & \\
& & & k_{n-1} & -k_{n} \\
& & & & k_{n}
\end{array}\right] \text {. }
$$




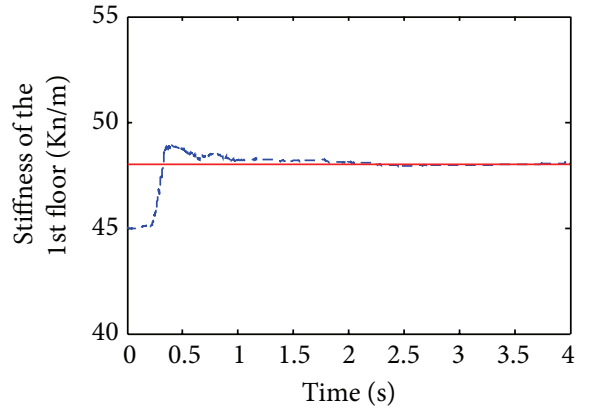

- - - Identified stiffness Real stiffness

(a) Stiffness of the 1st floor

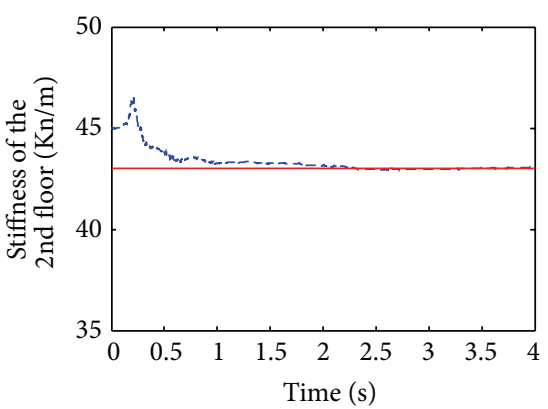

- - - Identified stiffness Real stiffness

(b) Stiffness of the 2nd floor

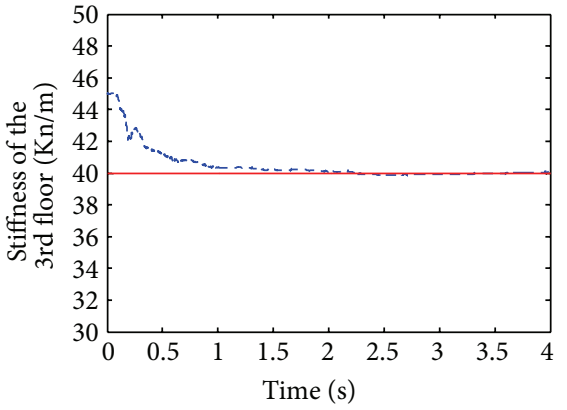

- - - Identified stiffness Real stiffness

(c) Stiffness of the 3rd floor

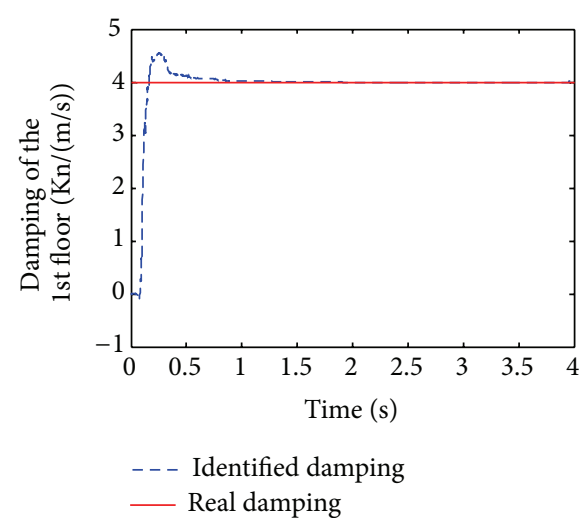

(d) Damping of the 1st floor

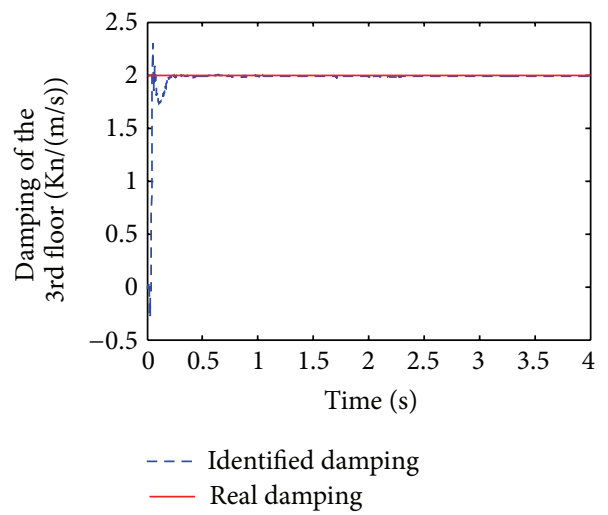

(f) Damping of the 3rd floor

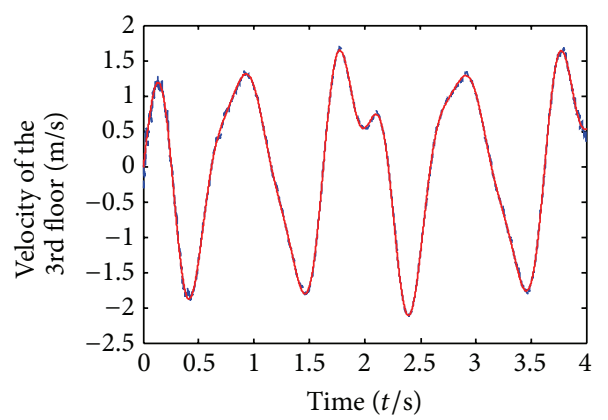

- - - Identified velocity

— Real velocity

(h) Velocity of the 3rd floor

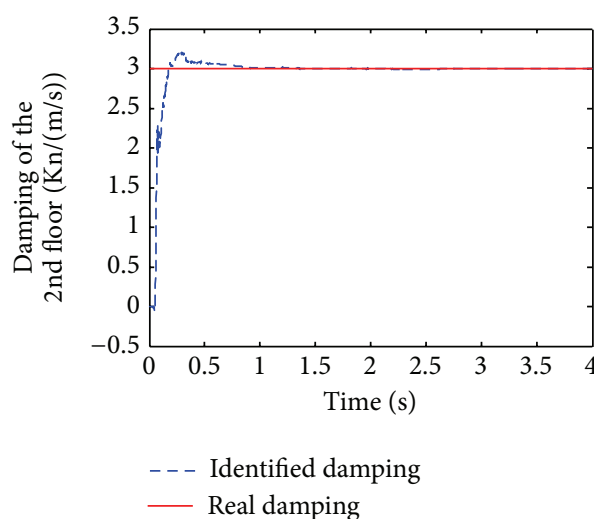

(e) Damping of the 2nd floor

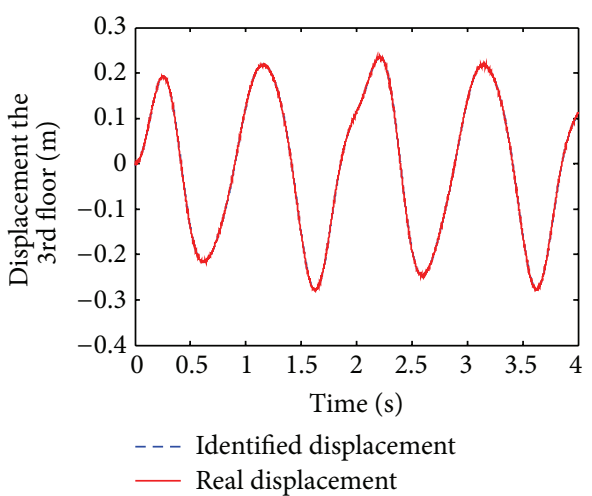

(g) Displacement of the 3rd floor

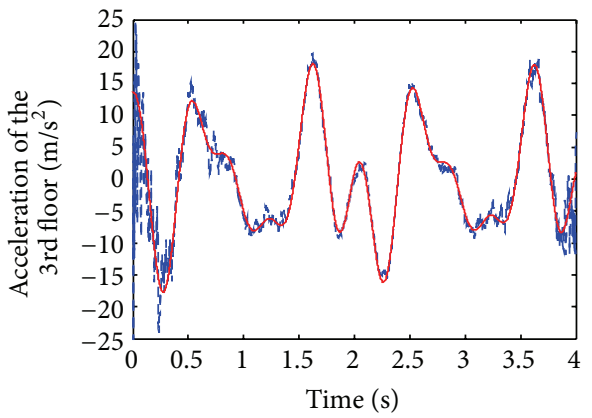

-.- Identified acceleration

— Real acceleration

(i) Acceleration of the 3rd floor

FIgure 2: Continued. 


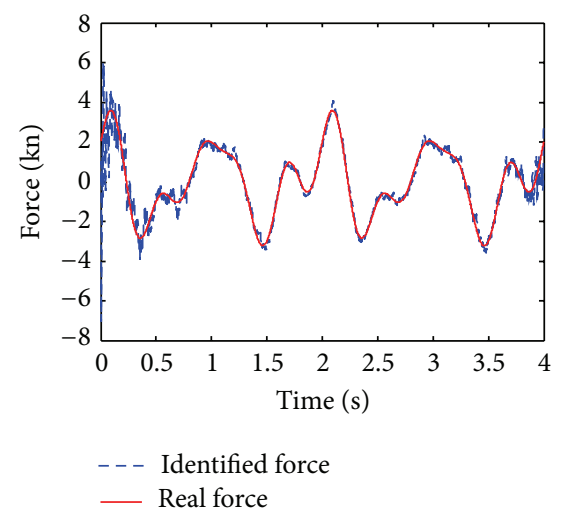

(j) External force

FIGURE 2: Identification results for linear structural system.

Beside $\mathbf{w}=\left[w_{1}, w_{2}, \ldots, w_{i}\right]^{T}, i=\left(1,2, \ldots, N_{f}\right)$, is the polynomial parameter vector. The responses $x_{i}(t)$ and $z_{i}(t)$ in (28) are the interstorey response and hysteretic component of $i$ th storey unit, respectively. In this paper $z_{i}(t)$ is defined as (5). $T_{m}^{i}$ is the orthogonal polynomial which is given by (20). The state vector is defined as

$$
\mathbf{Z}(t)=\left[x_{1 \sim n}, z_{1 \sim n}, \dot{x}_{1 \sim n}, k_{1 \sim n}, c_{1 \sim n}, \beta_{1 \sim n}, \gamma_{1 \sim n}, w_{1 \sim N_{m}}\right]^{\mathbf{T}} .
$$

Consider that $\dot{k}_{1 \sim n}=\dot{c}_{1 \sim n}=\dot{w}_{1 \sim N_{m}}=0$. Equation (28) can be written in state space as follows:

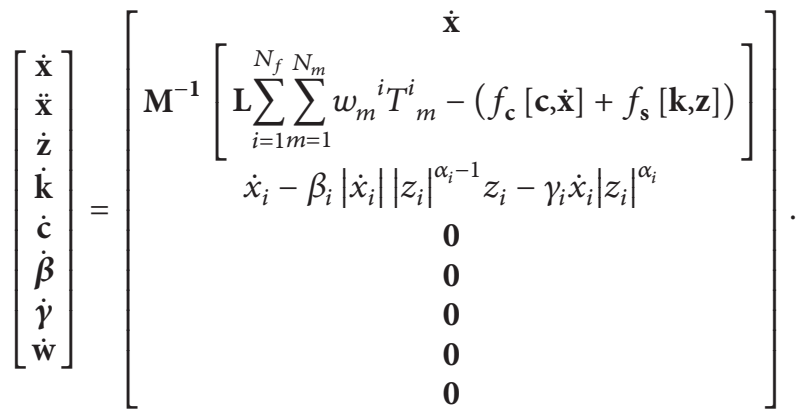

The observation equation is nonlinear in state space with the measurement of acceleration. The discrete linearized observation matrix can be calculated by (13).

\section{Implementation Procedure}

Step 1. Decompose structural excitation by using the Chebyshev standard orthogonal polynomial. The excitation is represented as orthogonal polynomial approximation. The accuracy of the approximation is according to the order of decomposition.

Step 2. Obtain the mass, damping, and stiffness matrices of the initial structural model, which may be inaccurate with model errors or initial structural damage.
Step 3. Conduct measurement on the structure. The "measured" data for the simulation studies is obtained from the solution of (1) or (6).

Step 4. Simultaneously identify structural parameter and polynomial parameters with the proposed EKF algorithm as shown in Section 3 from (12) to (19).

\section{Numerical Simulation Studies}

In this Section the proposed system identification method is validated numerically by the investigation of linear and nonlinear 3-storey shear frame as shown in Figure 1. The structural parameters and external force are simultaneously identified with incomplete contaminated measurement for the two cases. The sampling rate is $1000 \mathrm{~Hz}$ and 4-second measurements are collected for the identification.

7.1. Case 1: Three-Storey Linear Structure. A linear threestorey shear frame subject to $F(t)=\sin (4 \pi t)+2 \cos (2 \pi t)+$ $\sin (5 \pi t) \mathrm{kN}$ on the top of the floors is studied in this case. The equation of motion with excitation decomposition is shown in (25). The values of structural real parameters are $m_{1}=$ $m_{2}=m_{3}=500 \mathrm{~kg}, c_{1}=4 \mathrm{kNs} / \mathrm{m}, c_{2}=3 \mathrm{kNs} / \mathrm{m}, c_{3}=$ $2 \mathrm{kNs} / \mathrm{m}, k_{1}=48 \mathrm{kN} / \mathrm{m}, k_{2}=43 \mathrm{kN} / \mathrm{m}$, and $k_{3}=40 \mathrm{kN} / \mathrm{m}$. The horizontal displacements of all floors are measured. The "measured" displacements for this simulation are obtained from the solution of (1) with 5\% RMS noise process.

In this simulation, the mass of each floor is taken as known constant. The excitation history is decomposed by forty-order orthogonal polynomial. The unknown parameters are $k_{i}, c_{i}$ and $w_{j}(i=1,2,3$ and $j=1,2, \ldots, 40)$. The extended state vector is expressed as $\mathbf{Z}(t)=\left[x_{1-3}, \dot{x}_{1-3}\right.$, $\left.k_{1-3}, c_{1-3}, w_{1-40}\right]^{\mathbf{T}}$. The initial guess for $k_{i}, c_{i}$, and $w_{j}$ are $k_{i}=45 \mathrm{kN} / \mathrm{m}, c_{i}=0 \mathrm{kNs} / \mathrm{m},(i=1,2,3)$, and $w_{j}=0$ $(j=1,2, \ldots, 40)$. The initial guess of displacement and velocity in state variables are supposed as zero. Figure 2 shows the identified structural parameters and excitations. The stiffness and damping as shown in Figures 2(a)-2(f) are accurately identified. The value of the stiffness converges 


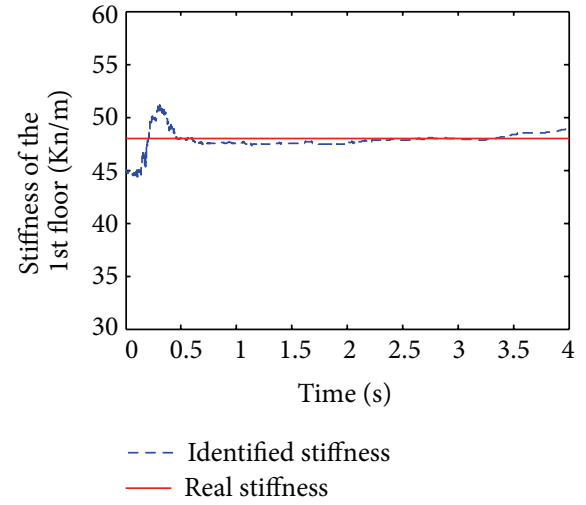

(a) Stiffness of the 1st floor

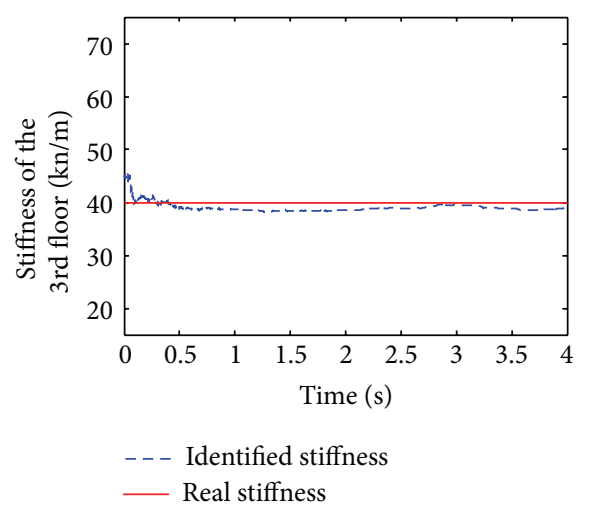

(c) Stiffness of the 3rd floor

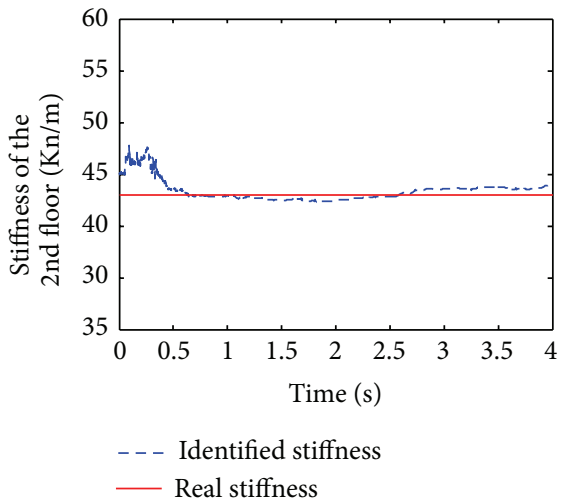

(b) Stiffness of the 2nd floor

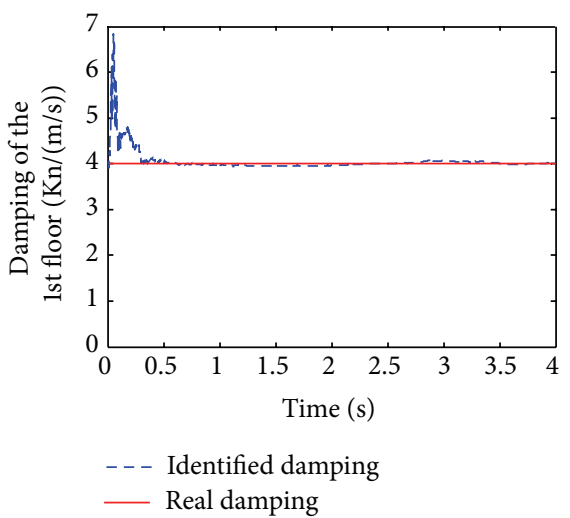

(d) Damping of the 1st floor

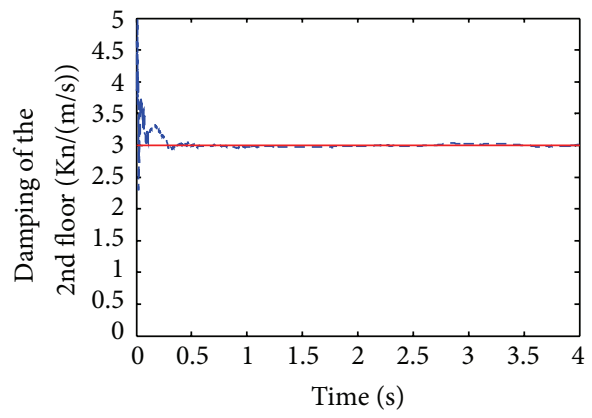

- - - Identified damping

- Real damping

(e) Damping of the 2nd floor

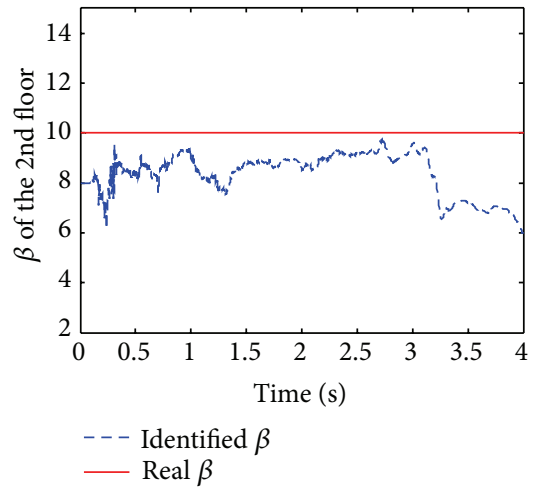

(h) $\beta$ of the 2nd floor

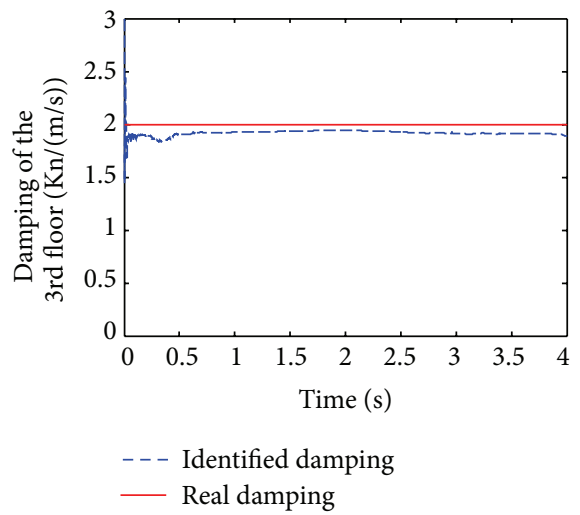

(f) Damping of the 3rd floor

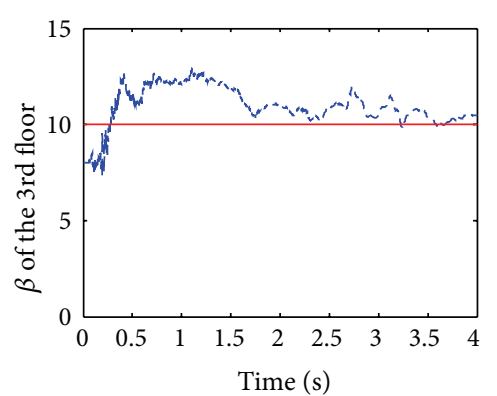

- - Identified $\beta$

(i) $\beta$ of the 3rd floor

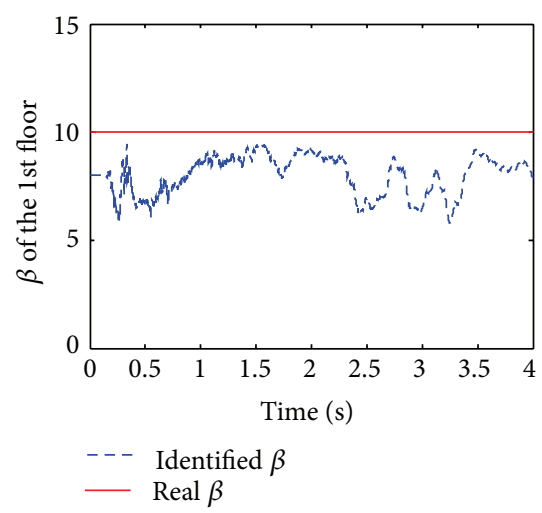

(g) $\beta$ of the 1st floor

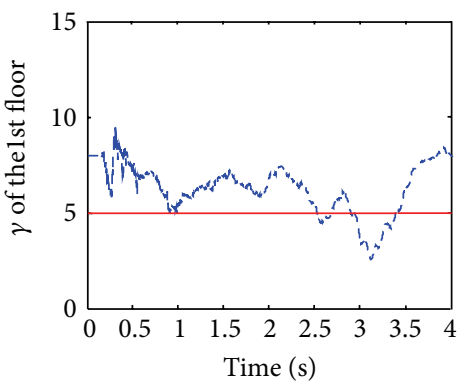

- - - Identified $\gamma$

— Real $\gamma$

(j) $\gamma$ of the 1st floor

Figure 3: Continued. 


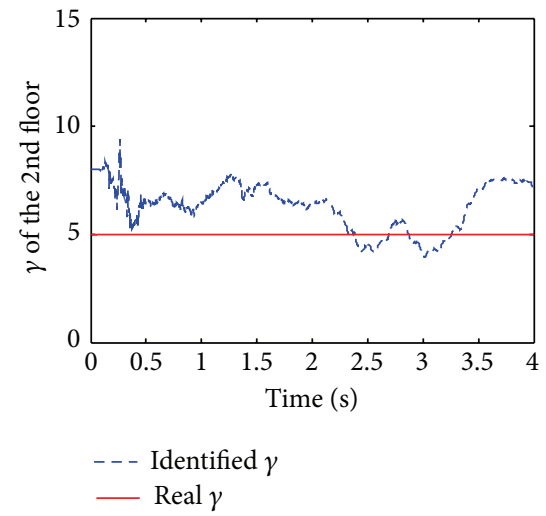

(k) $\gamma$ of the 2nd floor

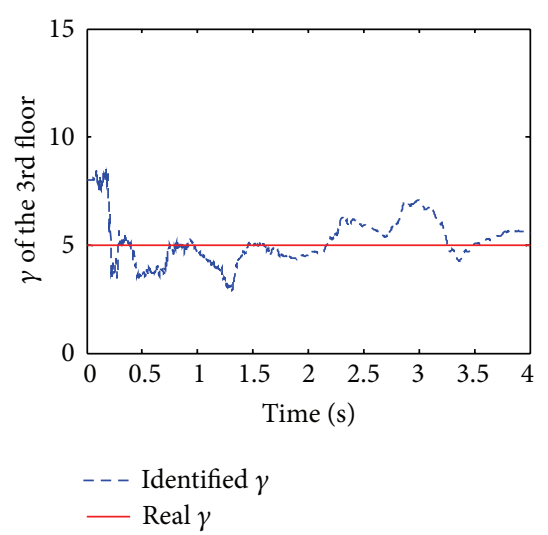

(l) $\gamma$ of the 3rd floor

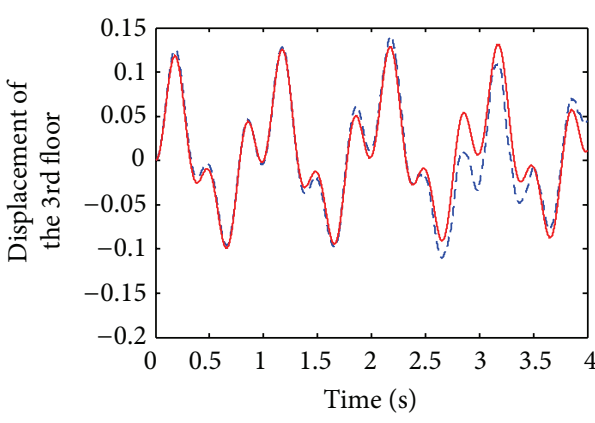

- - - Identified displacement

— Real displacement

(m) Displacement of the 3rd floor

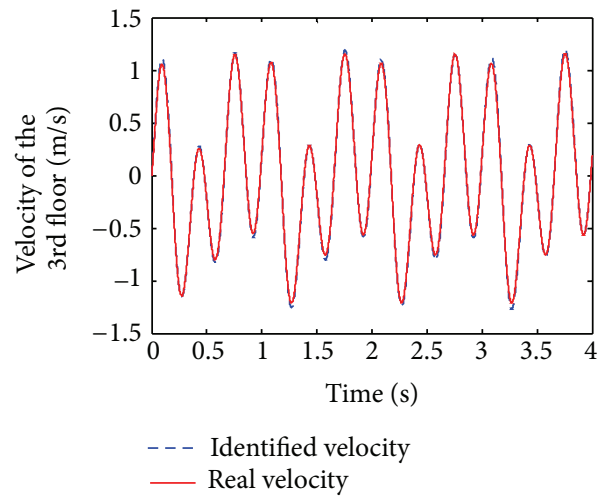

(n) Velocity of the 3rd floor

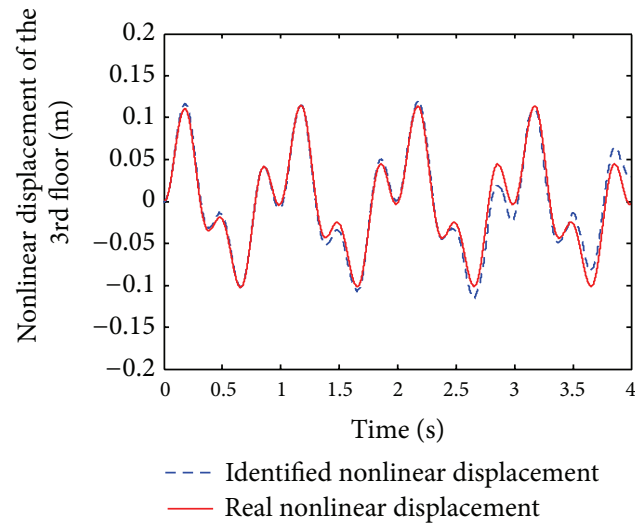

(p) Nonlinear displacement of the 3rd floor

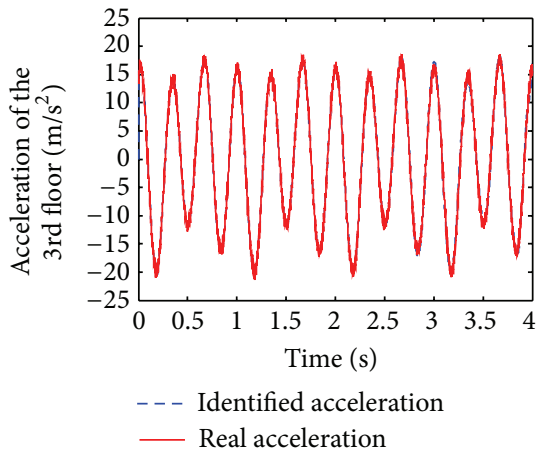

(o) Acceleration of the 3rd floor

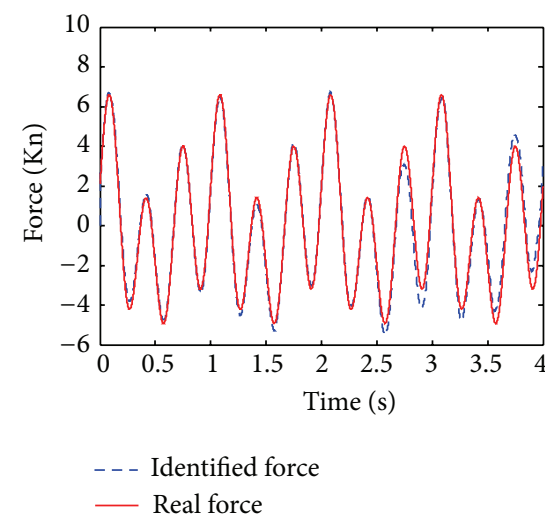

(q) External force

FIGURE 3: Identification results for nonlinear structural system.

to the real value in a short time even with the polluted measurement. In this study only the 3rd floor's displacement, velocity, and acceleration are listed as shown in Figures 2(g), 2(h), and 2(i). The identified and real displacement is nearly overlapping as shown in Figure $2(\mathrm{~g})$. There are some fluctuations at the beginning and the end of the time history of identified velocity though the identified velocity is very close to the real velocity as shown in Figure 2(h). The identified acceleration fluctuates severely at the beginning and the end compared to the real acceleration as shown in Figure 2(i). The displacement is identified with high accuratcy, while the acceleration is identified with lower accuracy. The force can also be identified with contaminated measurement with some fluctuations at the beginning and end of the time history. It is demonstrated that the structural parameter and excitation can be fairly accurately identified with contaminated measurement.

7.2. Three-Storey Hysteretic Nonlinear Structure. A threestorey hysteretic nonlinear shear frame subject to $F(t)=$ $4 \sin (6 \pi t)+2 \cos (2 \pi t)+\sin (4 \pi t) \mathrm{kN}$ on the top of the floors is investigated. The real values of the structure are 
$m_{1}=m_{2}=m_{3}=500 \mathrm{~kg}, c_{1}=4 \mathrm{kNs} / \mathrm{m}, c_{2}=3 \mathrm{kNs} / \mathrm{m}, c_{3}=$ $2 \mathrm{kNs} / \mathrm{m}, k_{1}=48 \mathrm{kN} / \mathrm{m}, k_{2}=43 \mathrm{kN} / \mathrm{m}, k_{3}=40 \mathrm{kN} / \mathrm{m}$, $\beta_{1}=\beta_{2}=\beta_{3}=10, \gamma_{1}=\gamma_{2}=\gamma_{3}=5$, and $n_{1}=$ $n_{2}=n_{3}=2$. The mass and parameter $n$ are also taken as known constant. The other parameters including stiffness, damping, and the parameters of Bouc-Wen model [19] are the unknowns to be identified. Only the horizontal acceleration of each floor is measured for the identification in this case study. The "measured" accelerations for this simulation are obtained from the solution of (4) with 5\% RMS noise. The excitation history is also decomposed by forty-order orthogonal polynomial. The unknown parameters are $k_{i}, c_{i}$, and $w_{j},(i=1,2,3$ and $j=1,2 \ldots, 40)$, the extended state vector is $\mathbf{Z}(t)=\left[x_{1-3}, \dot{x}_{1-3}, z_{1-3}, k_{1-3}, c_{1-3}, \beta_{1-3}, \gamma_{1-3}, w_{1-40}\right]^{\mathbf{T}}$, and the initial guess for $k_{i}, c_{i}, w_{m}, \beta_{i}$, and $\gamma_{i}$ is $k_{i}=45 \mathrm{kN} / \mathrm{m}$, $c_{i}=0 \mathrm{kNs} / \mathrm{m}, w_{m}=0$, and $\beta_{i}=\gamma_{i}=8$. The initial values of state variables are zero.

Figure 3 shows the identification results for the nonlinear structural system. Though there are a large number of fluctuations at the beginning of the identified time history, the stiffness and damping are fairly accurately identified with contaminated measurement as shown in Figures 3(a)3(f). The nonlinear parameters of Bouc-wen model are also identified with acceptable accuracy as shown from Figures $3(\mathrm{~g})$ to $3(\mathrm{l})$. The fluctuation at the beginning is also a little large in the time history of the nonlinear parameters, $\beta$ and $\gamma$. Similarly, in this case only the 3rd floor's displacement, nonlinear displacement, velocity and acceleration are listed as shown in Figures 3(m)-3(p). There are some errors in the amplitude of the identified displacement and nonlinear displacement. The identified velocity is nearly overlapping with the real velocity as shown in Figure 3(n). There are severe fluctuations in the peak of identified acceleration as shown in Figure 3(o), which is consistent with the linear case study. The external force is also identified accurately as shown in Figure 3(q). It is indicated that the system identification method with EKF and excitation decomposition can conduct the nonlinear structural system identification including structural parameter and external force with contaminated measurement.

\section{Conclusions}

A new method in time domain was proposed in this paper for simultaneous identification of structural parameters and loads based on limited output information with extended Kalman filter and orthogonal polynomials decomposition. The structural excitation is decomposed by orthogonal approximation. Then the structural parameters and coefficients of orthogonal polynomial are simultaneously identified with EKF. Numerical simulations of linear and nonlinear structures are utilized to study the effectiveness of the proposed method. From the simulation result, the proposed system identification method can conduct the structural system identification accurately and effectively with contaminated measurement. However, the severe nonlinear parameter, such as the nonlinear parameter $n$ of Bouc-Wen model, is taken as known in this paper. New identification method dealing with the severe nonlinear system identification will be developed in the future research.

\section{Conflict of Interests}

The authors declare that there is no conflict of interests regarding the publication of this paper.

\section{Acknowledgments}

The work described in this paper was supported by Project number 01319406 of Natural Scientific Research Innovation Foundation in Harbin Institute of Technology, Projects numbers 51161120360, 51308160, and 91315301 of National Natural Science Foundation of China, and Project 2013M541383 of China Postdoctoral Science Foundation.

\section{References}

[1] S. W. Doebling, C. R. Farrar, and M. B. Prime, "A summary review of vibration-based damage identification methods," Shock and Vibration Digest, vol. 30, no. 2, pp. 91-105, 1998.

[2] Y. Q. Ni, X. W. Ye, and J. M. Ko, "Monitoring-based fatigue reliability assessment of steel bridges: analytical model and application," Journal of Structural Engineering, vol. 136, no. 12, pp. 1563-1573, 2010.

[3] Y. Q. Ni, X. W. Ye, and J. M. Ko, "Modeling of stress spectrum using long-term monitoring data and finite mixture distributions," Journal of Engineering Mechanics, vol. 138, no. 2, pp. 175$183,2011$.

[4] G. W. Housner, L. A. Bergman, T. K. Caughey et al., "Structural control: past, present, and future," Journal of Engineering Mechanics, vol. 123, no. 9, pp. 897-971, 1997.

[5] Y. Zou, L. Tong, and G. P. Steven, "Vibration-based modeldependent damage (delamination) identification and health monitoring for composite structures-a review," Journal of Sound and Vibration, vol. 230, no. 2, pp. 357-378, 2000.

[6] J. Chen and J. Li, "Simultaneous identification of structural parameters and input time history from output-only measurements," Computational Mechanics, vol. 33, no. 5, pp. 365-374, 2004.

[7] Z. R. Lu and S. S. Law, "Identification of system parameters and input force from output only," Mechanical Systems and Signal Processing, vol. 21, no. 5, pp. 2099-2111, 2007.

[8] S. S. Law and D. Yong, "Substructure methods for structural condition assessment," Journal of Sound and Vibration, vol. 330, no. 15, pp. 3606-3619, 2011.

[9] Y. Ding, Structural control and condition assessment with substructure method [Ph.D. thesis], The Hong Kong Polytechnic University, 2012.

[10] H. Sun and R. Betti, "Simultaneous identification of structural parameters and dynamic input with incomplete output-only measurements," Structural Control and Health Monitoring, 2013.

[11] Y. Lei, C. Liu, and L. J. Liu, "Identification of multistory shear buildings under unknown earthquake excitation using partial output measurements: numerical and experimental studies," Structural Control and Health Monitoring, 2013.

[12] S. Haykin, A. H. H. Sayed, J. R. Zeidler, P. Yee, and P. C. Wei, "Adaptive tracking of linear time-variant systems by extended 
RLS algorithms," IEEE Transactions on Signal Processing, vol. 45, no. 5, pp. 1118-1128, 1997.

[13] J. N. Yang and S. Lin, "On-line identification of non-linear hysteretic structures using an adaptive tracking technique," International Journal of Non-Linear Mechanics, vol. 39, no. 9, pp. 1481-1491, 2004.

[14] G. Kerschen, K. Worden, A. F. Vakakis, and J.-C. Golinval, "Past, present and future of nonlinear system identification in structural dynamics," Mechanical Systems and Signal Processing, vol. 20, no. 3, pp. 505-592, 2006.

[15] G. Kerschen, M. Peeters, J. C. Golinval, and A. F. Vakakis, "Nonlinear normal modes, Part I: a useful framework for the structural dynamicist," Mechanical Systems and Signal Processing, vol. 23, no. 1, pp. 170-194, 2009.

[16] M. Peeters, R. Viguié, G. Sérandour, G. Kerschen, and J.-C. Golinval, "Nonlinear normal modes, Part II: toward a practical computation using numerical continuation techniques," Mechanical Systems and Signal Processing, vol. 23, no. 1, pp. 195216, 2009.

[17] H.-S. Tang, S.-T. Xue, R. Chen, and T. Sato, "Online weighted LS-SVM for hysteretic structural system identification,” Engineering Structures, vol. 28, no. 12, pp. 1728-1735, 2006.

[18] J. N. Yang, S. Pan, and S. Lin, "Least-squares estimation with unknown excitations for damage identification of structures," Journal of Engineering Mechanics, vol. 133, no. 1, pp. 12-21, 2007.

[19] Y. K. Wen, "Equivalent linearization for hysteretic systems under random excitations," Journal of Applied Mechanics, Transactions ASME, vol. 47, no. 1, pp. 150-154, 1980. 


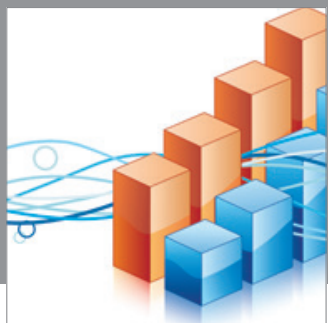

Advances in

Operations Research

mansans

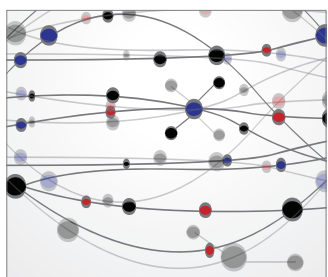

The Scientific World Journal
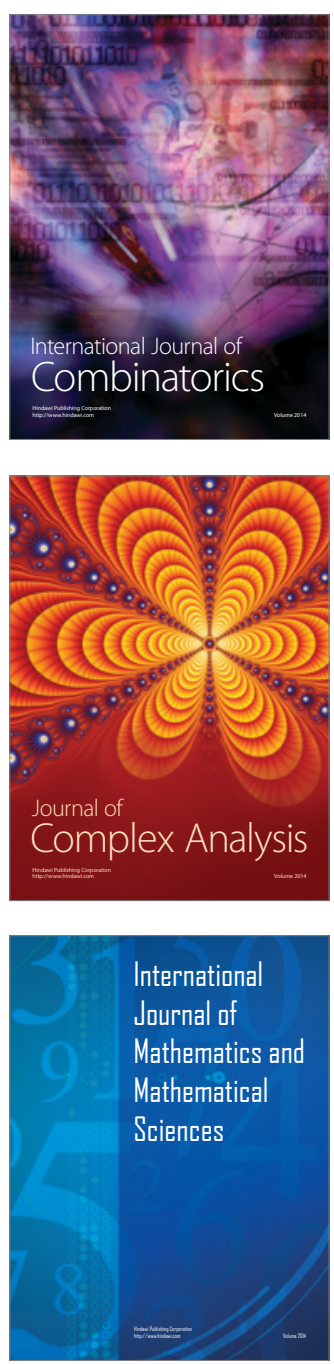
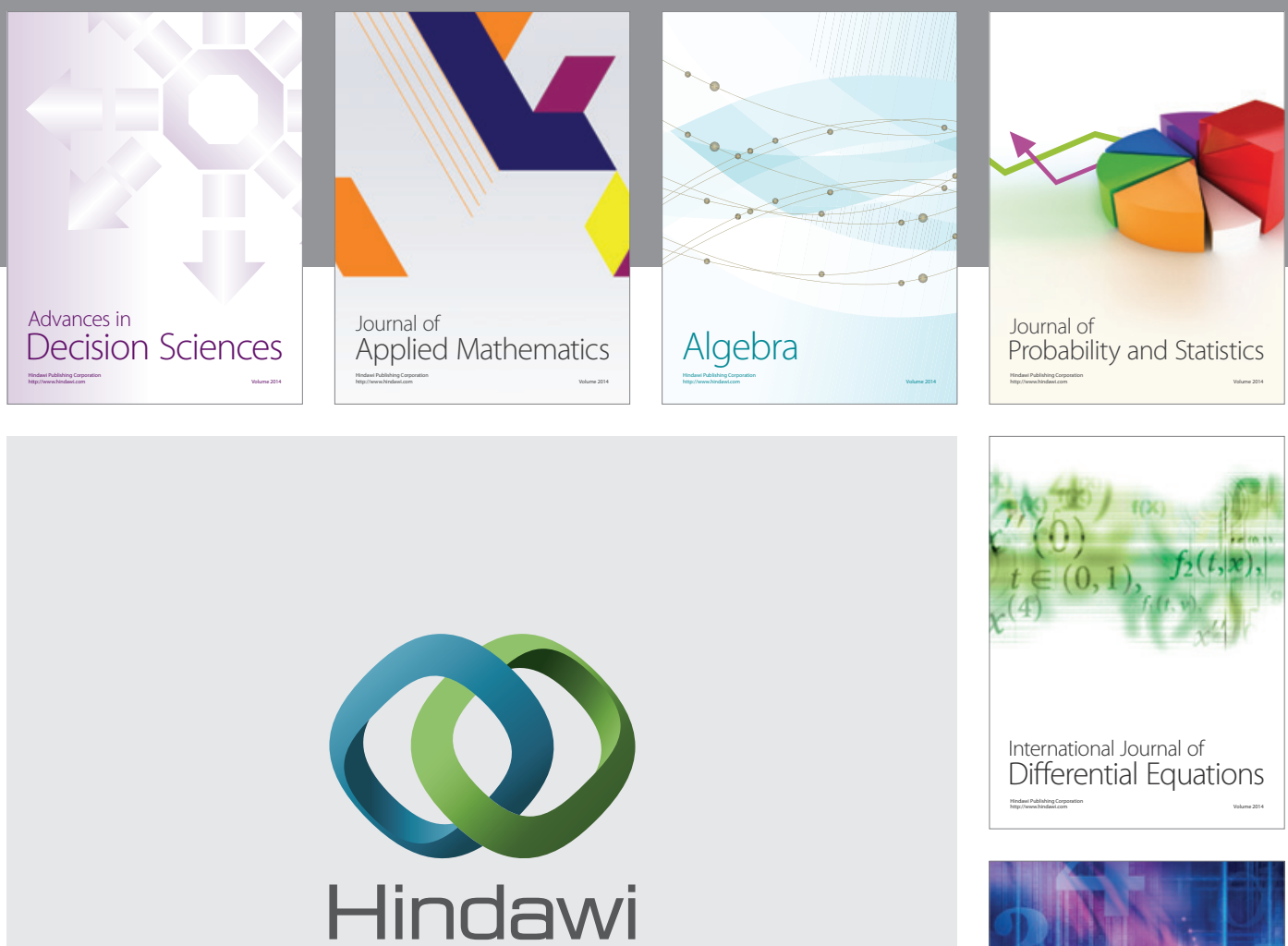

Submit your manuscripts at http://www.hindawi.com
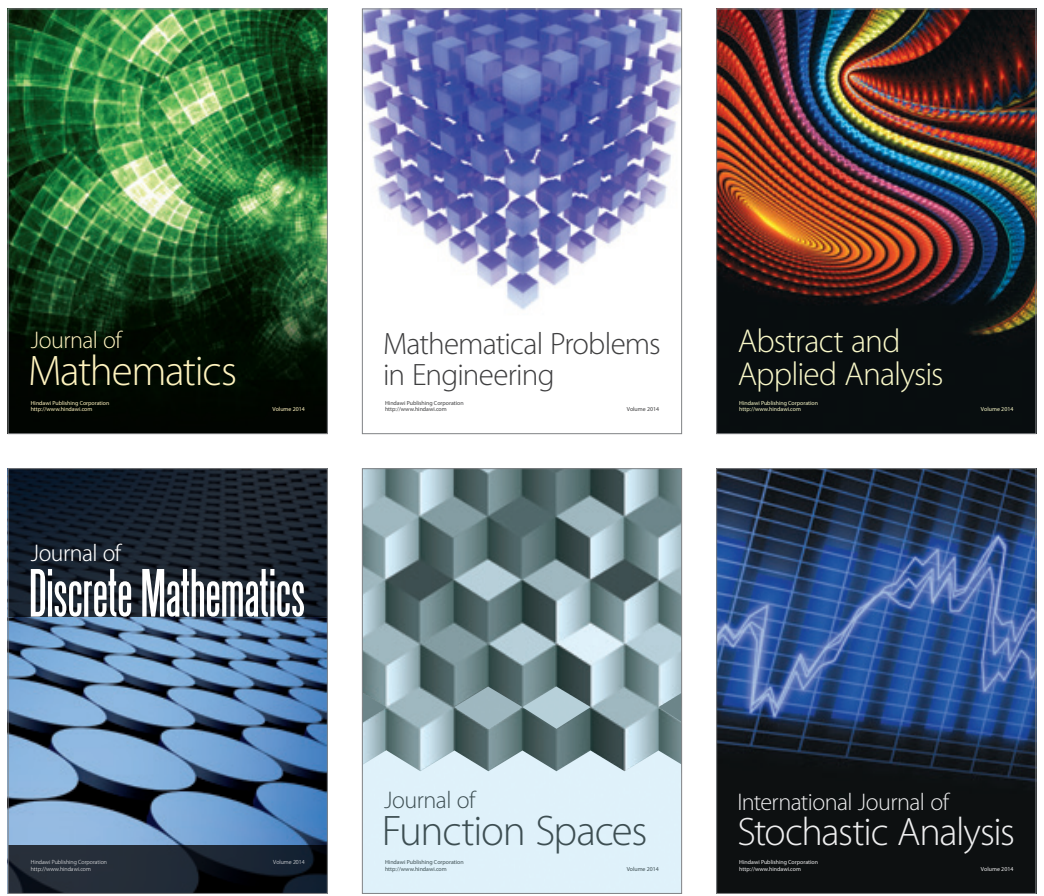

Journal of

Function Spaces

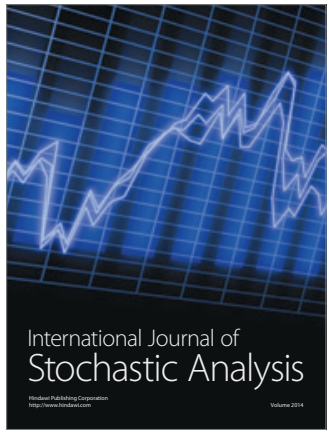

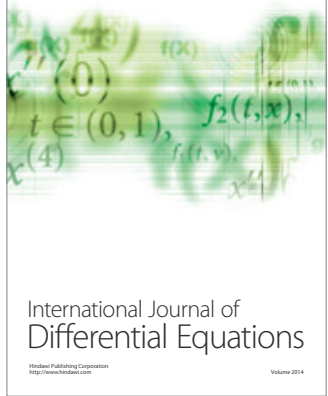
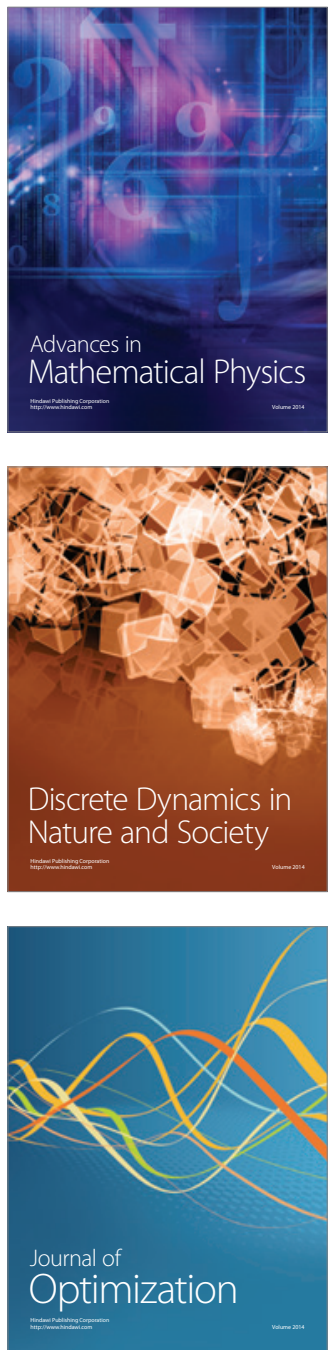